\title{
Effects of noise letters on decisions: Discrete or continuous flow of information?
}

\author{
JEFF MILLER \\ University of California, San Diego, La Jolla, California 92903
}

\begin{abstract}
Four forced-choice letter-detection experiments examined the effect on detection latency of noise letters that were visually similar to target letters. A single target letter was present in each display. Noise letters were similar to the target letter present in the display (the signal), to a different target letter assigned to the same response as the signal, or to a target letter assigned to the opposite response from the signal. Noise letters were present in either relevant or irrelevant display positions, and two quite different stimulus sets were used. The experiments were designed to test a prediction of models in which information about noise letters is transmitted continuously from the recognition to the decision process. These models predict that responses should be faster when noise letters are visually similar to a target assigned to the same response as the signal than when noise letters are similar to a target assigned to the opposite response. Statistically reliable effects of the type predicted by continuous models were obtained when noise letters appeared in relevant display positions, but not when they appeared in irrelevant positions.
\end{abstract}

The mental processing occurring between stimulus presentation and choice response is regarded by virtually all theories of choice reaction time (RT) as a composition of several conceptually distinct subprocesses (cf. Smith, 1968). There is considerable agreement about the general functions of some of these processes, enunciated at least as early as Donders (1868). A stimulus must be perceived, or encoded, to the extent necessary for whatever discriminations are required by the task (recognition). Given information about what stimulus was presented, a choice must be made as to what course of action is required (decision). Finally, at least in the logical sequence, motor commands needed to perform the appropriate action must be prepared and initiated (response).

While there is consensus about some of the functions required to perform choice RT tasks, there is debate about the temporal relations among processes responsible for these functions. Two extreme views, referred to here as the "discrete" and "continuous" views, can be distinguished. According to the discrete view, different functions are carried out in strict sequence, with each process beginning only after the previous process has terminated (e.g., Broadbent, 1958; Sperling, 1967; Sternberg, 1969a, 1969b). According to the continuous view, on the other hand, different processes may be active at the same time

I would like to thank Diane Fisher and Eureka Harami, for their assistance in collecting the data, and David Bauer and Greg Stone, for helpful comments on a previous draft of the manuscript. Financial support for this project was provided by NSF Grant BNS-7824772. Requests for reprints should be addressed to the author at the Department of Psychology, C-009, UCSD, La Jolla, California 92903. (e.g., Norman \& Bobrow, 1975). The logical sequence of the recognition, decision, and response operations is preserved in continuous models, because the output of one process serves as the input to the next. However, continuous models allow processes to transmit partial information, enabling one process to begin before the previous one is completely finished.

The experiments reported in this paper were designed to study the transmission of information from the recognition process to the decision process. The goal was to obtain evidence relevant to the question of whether the decision process begins before the identification process is completely finished. These experiments incorporate an elaboration of techniques used extensively by $C$. W. Eriksen and his co-workers (e.g., B. A. Eriksen \& C. W. Eriksen, 1974; C. W. Eriksen \& Schultz, 1979). C. W. Eriksen and Schultz (1979) summarized a variety of results from this research program, and they described a continuous flow model that accounts for the results quite easily. ${ }^{1}$ The experiments reported in this article test a prediction of their continuous flow model in an experimental condition not previously examined.

The basic visual search paradigm used by Eriksen and his colleagues involved linear displays of letters, with one relevant signal letter centered over fixation and several irrelevant noise letters appearing to either side. One or more target letters were assigned to each of two responses (e.g., press the right button if signal letter is $\mathrm{C}$ or I and press the left button if it is $\mathrm{H}$ or $\mathrm{M}$ ). Subjects made a forced-choice response to indicate target set membership of the signal letter. One major experimental question concerned the effect of using letters from one of the target sets as noise letters. Large consistency effects have always been obtained. 
On "signal consistent trials," when noise letters are identical to the signal letter (e.g., CCCCC), responses are faster and more accurate than a control condition with neutral irrelevant letters. Responses are also faster and more accurate than control on "response consistent trials," when noise letters are identical to a target letter assigned to the same response as the signal (e.g., IICII). On "response inconsistent trials," when noise letters are identical to a target letter assigned to the other response (e.g., $\mathrm{HHCHH}$ ), responses are slower and less accurate than control.

As C. W. Eriksen and Schultz (1979) noted, consistency effects can easily be interpreted in terms of a continuous activation model. Basically, they suggested a multilevel processing model, with information about stimuli accumulating continuously at all levels within the system (cf. McClelland \& Rumelhart, 1981). Initially, crude visual information activates each response to the extent that it is consistent with that response. Later, with increasing stimulus specification, activation of the correct response comes to dominate. Activation contributed by noise letters has a priming effect, so responses are faster when noise letters prime the appropriate response than when they prime the competing response. Occasionally, activation contributed by the noise letters may be sufficient to trigger a response, so there are also more errors in the response-inconsistent condition than in the signal or response-consistent conditions.

Consistency effects can also be obtained with noise letters visually similar (VS) to targets rather than identical (ID) to targets. B. A. Eriksen and C. W. Eriksen (1974) found that responses were facilitated when noise letters were visually similar to the signal letter ("VS signal consistent" trials). However, responses were inhibited when noise letters were visually similar to target letters assigned to the opposite response ("VS response inconsistent" trials). Naturally, both effects were somewhat smaller than the corresponding effects for ID signal-consistent and ID responseinconsistent trials. They did not include a condition with noise letters dissimilar to the signal but similar to another target assigned to the same response as the signal ("VS response consistent" trials).

Continuous models explain consistency effects obtained with noise letters visually similar to targets in terms of transmission of information about partially activated target letters (cf. McClelland \& Rumelhart, 1981). For example, suppose that $C$ was a target letter and $G$ was not. In most type fonts, presenting $G$ as a noise letter would increase the level of activation of the internal code for the letter $C$. Under normal viewing conditions, this increased activation would be both small and short-lived. Subsequent perceptual processing would establish the presence of the $G$, and the activation corresponding to the $\mathrm{C}$ would fade quickly. However, in continuous models, the partial activation of $\mathrm{C}$ would already have been transmitted to the decision process before the recognition of $G$ was complete. This activation could prime the decision associated with $\mathrm{C}$ and thereby influence the overall decision latency. Decisions would thus be faster when noise letters partially activated response-consistent targets than when they partially activated response-inconsistent targets.

The continuous flow mechanism would also produce response facilitation when noise letters were visually similar to a target letter different from the signal but assigned to the same response as the signal ("VS response consistent" trials). For example, suppose that $\mathrm{C}$ and I were two targets assigned to the right-hand response, and that a stimulus display contained $\mathrm{C}$ as a signal surrounded by $\mathrm{Ts}$ as noise letters. According to the continuous flow conception, partial output from any response-consistent letter should speed the response, even if this letter is not the same as the signal letter. In this example, the Ts should partially activate the code for I, which, in turn, would activate the decision to respond on the right. Thus, a noise letter visually similar to a responseconsistent target should produce faster responses than a noise letter visually similar to a responseinconsistent target.

The purpose of the present experiments was to test the prediction that responses should be faster when a noise letter is visually similar to a response-consistent target than when a noise letter is similar to a responseinconsistent target. It is possible to make the comparison of interest for noise letters in both relevant and irrelevant display positions, because none of the critical noise letters are themselves targets. In order to maximize contact with prior work, the first experiment made the comparison in a forced-choice letter-detection task, with the center position designated relevant and the flanker positions designated irrelevant. In the second experiment, however, two display positions were used, with both relevant to the decision. The third and fourth experiments applied the same criterion for continuous output to a letterdetection task with large letters made out of small letters (cf. Navon, 1977), again varying whether the noise letters appeared in relevant or irrelevant display positions. In outline, then, the four experiments of this paper were a factorial combination of two stimulus sets, with noise letters appearing in relevant or irrelevant display positions.

\section{EXPERIMENT 1}

\section{Method}

Apparatus and Stimuli. Stimulus presentation and recording of responses were controlled by an Automated Data Systems Micro-8 computer. Stimuli were the 10 uppercase letters, C, G, I, T, U, V, $K, R, M$, and $D$. The first 8 of these letters constitute four pairs of visually similar letters in the particular font used, and the last 2 letters were selected as neutral letters relatively dissimilar to the other eight. The visual similarity of the letters within pairs (CG, 
IT, UV, and KR) was established in previous work with the same stimuli and apparatus (Miller \& Bauer, 1981).

Stimulus letters appeared as light solid line drawings on the dark background of an oscilloscope and were viewed from a distance of about $50 \mathrm{~cm}$. Individual letters subtended visual angles of about $.36 \mathrm{deg}$, as did the spaces between letters. Responses were made with the left and right index fingers on a microswitch keyboard located directly in front of the subject.

Subjects and Procedure. Subjects were 32 undergraduates recruited on the San Diego campus of the University of California. Subjects were informed that they would be presented with horizontal arrays of three letters, and they were instructed to respond only to the center letter, ignoring the two flanking letters. The two noise letters were always identical to each other. In order to help subjects focus their attention on the relevant letter position, a fixation point was displayed briefly in this location $350 \mathrm{msec}$ before stimulus onset.

For each subject, all eight letters in the four visually similar pairs were used, as well as one of the two neutral letters. One target letter was selected from each of the four pairs of visually similar letters. Of the four letters thus selected, two were assigned to one response button and two were assigned to the other response button. Different letter selections were made for each subject, and the assignment of letters to conditions was counterbalanced across subjects. On each trial, one of the four target letters appeared as the signal in the relevant, center position of the display. These letters also appeared as noise letters in some conditions. The four letters visually similar to the target letters appeared only as noise letters. In all, there were 36 different stimulus conditions defined by four possible signal letters, each of which could occur with any of nine possible noise letters (four letters identical to targets + four letters visually similar to targets + one neutral letter).

Each subject was tested in two blocks of 150 trials. The first 6 trials in each block were considered practice and were not recorded. The remaining 144 trials were divided equally among the 36 different signal/noise conditions. Thus, each signal letter appeared four times with each possible noise letter.

\section{Results and Discussion}

Average RT and percentage of errors (PE) were computed for the seven logically distinct conditions defined by the relationship of noise letters to signal letter, and averages across subjects are shown in Table 1.

For both RT and PE, analyses of variance were computed with factors of noise condition, block, and subjects. In the analysis on PE, only the effect of noise condition was statistically reliable $[F(6,186)=$ 3.69, $\mathrm{p}<.05, \mathrm{MSe}=24.2$ ]. Pairwise comparisons can be made using the least significant difference of $1.75 \%$. Of major interest, for present purposes, is that there were no reliable differences among the conditions with noise letters visually similar to targets.

Results of the analysis of variance on RT were considerably more complicated. Again, the main effect of noise condition was reliable $[\mathrm{F}(6,186)=6.96$, $\mathrm{p}<.05, \mathrm{MSe}=1,869]$. Pairwise comparisons can be made using a least significant difference of $15.1 \mathrm{msec}$. In addition, the interaction of block $\times$ noise condition was also reliable $[F(6,186)=2.94, p<.05$, $\mathrm{MSe}=2,007]$. Post hoc testing of this interaction revealed that it was partly due to a greater practice effect with signal and noise letters identical (ID signalconsistent condition) than in any other condition. Furthermore, practice effects in the VS signal-consistent and VS response-consistent conditions were less than the average practice effect across the other conditions.

Of particular interest for the prediction of continuous flow models is the comparison between VS response-consistent and VS response-inconsistent trials. Though there was a $22-\mathrm{msec}$ difference in the direction predicted by continuous models in the first block, the effect in this block was short of statistical significance, even with the liberal LSD test $(.05<\mathrm{p}$ $<.06$ ). Moreover, in the second block, the advantage for the VS response-consistent condition disappeared almost completely. Thus, while the results hint at the result predicted from continuous flow models early in practice, it is not possible to say that they demonstrate it unequivocally.

These data are also relevant to the issue of how recognition of one letter is influenced by the presence in the display of another letter with identical or similar features (e.g., Krueger \& Shapiro, 1980). Responses were faster when noise letters were identical to the signal than when they were identical to response consistent targets. Since these two conditions were equated with respect to decision effects, the difference between them suggests that identity of the noise letters to the signal speeds recognition of the signal. This result is consistent with a variety of findings showing improved speed and accuracy of

Table 1

Experiment 1: RT and PE as a Function of Noise Condition and Experimental Block

\begin{tabular}{|c|c|c|c|c|c|c|c|}
\hline \multirow{2}{*}{$\begin{array}{c}\text { Sample Stimulus } \\
\text { Display }\end{array}$} & \multirow[b]{2}{*}{ Noise Condition } & \multicolumn{2}{|c|}{ Block 1} & \multicolumn{2}{|c|}{ Block 2} & \multicolumn{2}{|c|}{ Average } \\
\hline & & $\mathrm{RT}$ & PE & RT & PE & RT & PE \\
\hline M C M & Neutral & 685 & 5.9 & 669 & 3.4 & 677 & 4.7 \\
\hline $\mathrm{CCC}$ & ID Signal Consistent & 682 & 3.2 & 626 & 2.8 & 654 & 3.0 \\
\hline I C I & ID Response Consistent & 665 & 2.6 & 638 & 2.4 & 652 & 2.5 \\
\hline $\mathrm{UCU}$ & ID Response Inconsistent & 695 & 6.1 & 682 & 5.7 & 689 & 5.9 \\
\hline G C G & VS Signal Consistent & 680 & 3.8 & 679 & 2.4 & 680 & 3.1 \\
\hline $\mathbf{T C T}$ & VS Response Consistent & 657 & 2.6 & 658 & 3.6 & 658 & 3.1 \\
\hline V C V & VS Response Inconsistent & 679 & 3.9 & 662 & 4.3 & 671 & 4.1 \\
\hline
\end{tabular}

Note-Sample stimulus displays refer to target sets of $C I$ assigned to one response and $U K$ assigned to the other. ID $=i d e n t i c a l$ to target letter; $V S=$ visually similar to target letter. 
letter recognition when several identical letters are presented in a display (C. W. Eriksen, 1966; C. W. Eriksen \& B. A. Eriksen, 1979; C. W. Eriksen \& Lappin, 1965; but see Krueger \& Shapiro, 1980). On the other hand, the VS signal-consistent condition was reliably slower than the VS response-consistent condition. By the same logic, this finding suggests that visual similarity of noise letters to the signal slows recognition of the signal. This conclusion is consistent with the interactive channels model of Estes (1974), as well as experimental results indicating that recognition accuracy with tachistoscopic presentations is slightly impaired when an identical or visually similar noise letter is presented together with a signal letter (Bjork \& Murray, 1977; Santee \& Egeth, 1980). Taken together, the two apparently discrepant findings of this study support the view that similarity of noise letters to the signal may have inhibitory effects at one level and facilitatory effects at another (cf. C. W. Eriksen \& B. A. Eriksen, 1979; Santee \& Egeth, 1980). In particular, feature analysis may be a somewhat different process from activating an abstract letter code (Mewhort, Campbell, Marchetti, \& Campbell, 1981). It is possible, then, that similar letters interfere with feature analysis, possibly because of competition for feature-specific channels (Bjork \& Murray, 1977; Estes, 1974). On the other hand, two identical letters may jointly activate the same letter code, facilitating the recognition of that letter. This coactivation could more than make up for featural inhibition when the two letters are identical, yielding a net facilitation. When letter recognition is data-limited, as in the tachistoscopic studies of Bjork and Murray (1977), a small effect on feature analysis would be crucial, since it could prevent successful recognition. When recognition is resource-limited, as in RT studies (B. A. Eriksen \& C. W. Eriksen, 1974; C. W. Eriksen \& Schultz, 1979), a small inhibition of feature analysis would only produce a slight delay, which might be overcome by a larger facilitation at another point in processing. Under the present stimulus conditions, apparently, inhibitory effects outweighed facilitatory effects only with noise letters visually similar to the signal. It should also be emphasized that the results do not replicate B. A. Eriksen and C. W. Eriksen's (1974) finding of an advantage, predicted by continuous flow models, for VS signalconsistent trials over VS response-inconsistent trials.

It is also of interest to consider the practice effects observed in this experiment. When noise letters were identical to target letters, only the second block showed the usual ordering of RTs: ID signal consistent, ID response consistent, neutral, and ID response inconsistent. In the first block, the ID signalconsistent condition was unusually slow. Since previous studies have not reported data from the start of practice (e.g., C. W. Eriksen \& Schultz, 1979), this cannot be considered a failure to replicate their results. The finding does have interesting implications regarding what it is that subjects learn in this task. Apparently, subjects do not have great difficulty developing an inhibition of irrelevant letter positions. Instead, the slow ID signal-consistent condition indicates that inhibition of the noise letters is quite strong in the first block. It looks like subjects learn to relax this inhibition somewhat. This enables them to take advantage of consistent information without making too many mistakes on inconsistent trials.

\section{EXPERIMENT 2}

The results of the first experiment did not unambiguously confirm the predictions of the continuous model. VS response-inconsistent trials were not reliably slower than either VS response-consistent trials or VS signal-consistent trials. On the other hand, some suggestion of continuous output was obtained early in practice, and it would be quite premature to conclude that output is discrete without considering other experimental procedures designed to maximize the effect of continuous output. As noted earlier, the comparisons of interest can also be made in a task with all display positions relevant. Putting noise letters in relevant positions should at least exaggerate any effects they produce, and may also bring about qualitative changes in the way they are processed.

As in the previous experiment, two target letters were assigned to each of two responses. A single signal letter and a single noise letter appeared in each stimulus display. The noise letter was always similar to one of the target letters: the presented signal letter (VS signal-consistent condition), the other target assigned to the same response as the signal (VS responseconsistent condition), or a target letter assigned to the opposite response from the signal (VS responseinconsistent condition). Since both display positions were relevant, no trials with noise letters identical to targets could be included. As in the previous experiment, the question of major interest was whether VS response-consistent trials would be faster than VS response-inconsistent trials.

\footnotetext{
Method

The apparatus and stimuli were the same as those used in Experiment 1 , except that the neutral letters were not used. Thus, each subject had two target letters assigned to one response button and two target letters assigned to the other response button. On each trial, one of these four target letters was displayed, together with a single noise letter that was a letter visually similar to one of the four target letters. Letters and spaces between letters were the same size as in Experiment 1.

Each of 36 subjects was tested in two blocks of 164 trials. The first 4 trials in each block were considered warm-up, and were not recorded. The remaining 160 trials were equally divided among 32 conditions. Each of the four target letters could appear as
} 
the signal, the signal could appear on the left or the right side of the display, and the noise letter could be visually similar to any of the four target letters.

\section{Results and Discussion}

For each subject and block, average $\mathrm{RT}$ and PE were recorded as a function of three experimental factors: signal side, response hand, and noise condition. Table 2 shows overall average RT and PE for the response hand $x$ noise condition interaction. Evidence for continuous output was obtained with both hands, in that VS response-consistent trials were faster than VS response-inconsistent trials.

An analysis of variance on RT was performed with factors of subjects, blocks, response hand, signal side, and noise condition. This analysis confirmed the significance of the main effect of noise condition $[\mathrm{F}(2,70)=24.4, \mathrm{p}<.01, \mathrm{MSe}=4,899]$. Planned comparisons indicated significant differences $(p<$ .01) between all pairs of means on this factor. In addition, responses were $43 \mathrm{msec}$ faster in the second block than in the first $[\mathrm{F}(1,35)=6.5, \mathrm{p}<.01, \mathrm{MSe}=$ $62,307]$, and responses were $23 \mathrm{msec}$ faster when the signal letter appeared on the left side of the display than when it appeared on the right side $[F(1,35)=$ $20.4, \mathrm{p}<.01, \mathrm{MSe}=5,753$ ].

Two interactions were significant in the analysis of RTs. First, the response hand $\times$ signal side interaction was reliable $[\mathrm{F}(1,35)=6.16, \mathrm{p}<.01, \mathrm{MSe}=$ $7,910]$, due to a greater advantage for the right hand when the signal appeared on the right side of the display than when it appeared on the left side (cf. Simon, 1969). Second, the response hand $x$ noise condition interaction shown in Table 2 was also significant $[F(2,70)=4.42, p<.025, \mathrm{MSe}=7,373]$. Although the left hand was about 29 msec slower than the right on VS response-consistent and VS response-inconsistent trials, the left hand was slightly faster on VS signal-consistent trials. Inspection of the error rates suggests that some sort of bias may account for this interaction. Accuracy was about $1.7 \%$ higher for the left hand than for the right hand on VS response-consistent and response-inconsistent trials, while it was $1.4 \%$ higher for the right hand on VS signal-consistent trials. For some reason, then,

Table 2

Experiment 2: RT and PE as a Function of Noise Condition and Response Hand

\begin{tabular}{|c|c|c|c|c|c|c|}
\hline \multirow[b]{3}{*}{ Noise Condition } & \multicolumn{6}{|c|}{ Response Hand } \\
\hline & \multicolumn{2}{|c|}{ Left } & \multicolumn{2}{|c|}{ Right } & \multicolumn{2}{|c|}{ Average } \\
\hline & RT & PE & RT & PE & RT & PE \\
\hline $\begin{array}{l}\text { VS Signal Consistent } \\
\text { VS Response Consistent } \\
\text { VS Response Inconsistent }\end{array}$ & $\begin{array}{l}724 \\
766 \\
782\end{array}$ & $\begin{array}{l}3.3 \\
3.2 \\
6.9\end{array}$ & $\begin{array}{l}732 \\
737 \\
754\end{array}$ & $\begin{array}{l}1.9 \\
5.0 \\
8.5\end{array}$ & $\begin{array}{l}728 \\
752 \\
768\end{array}$ & $\begin{array}{l}2.6 \\
4.1 \\
7.7\end{array}$ \\
\hline
\end{tabular}

Note $-V S=$ visually similar to target letter. it appears that subjects were more cautious on VS signal-consistent trials when the signal was assigned to the right hand than when it was assigned to the left.

An analysis of variance was also performed on PE, with factors identical to those in the analysis of RT. There were reliably fewer errors in the second block than in the first $[4.2 \%$ vs. $5.4 \% ; F(1,35)=5.78$, $\mathrm{p}<.01, \mathrm{MSe}=56.7]$. The effect of noise condition was also reliable $[F(2,70)=7.4, p<.01, M S e=265.6]$, with significantly lower accuracy on VS responseinconsistent trials than on VS response-consistent trials or VS signal-consistent trials. No other main effects or interactions were statistically reliable.

Overall, the results of Experiment 2 confirm the prediction based on continuous transmission of information from the recognition to the decision process. Looking only at conditions in which noise letters were visually dissimilar to signal letters, there was an advantage when noise letters were similar to a response-consistent target letter as opposed to a response-inconsistent target letter. This effect was predicted by continuous output models, because noise letters would produce partial activation in target codes relevant to the response. Though these codes would not be fully activated (i.e., target letters are not incorrectly recognized), they do seem to contribute some activation to the decision. It appears that partial activations in the recognition process contribute to decisions and that information about activations is transmitted from recognition to decision processes before these activations reach the levels needed for discrete recognition.

Since noise letters were visually similar to target letters, it is necessary to consider the possibility that subjects occasionally mistakenly recognized noise letters as target letters. Though the continuous model explains the consistency effect for visually similar letters by means of partial activations produced in codes corresponding to target letters, misidentifications might have produced the consistency effect through complete activation of these codes. If the subject mistakenly perceived two targets in the same display, RT might be influenced by whether these were consistent or inconsistent targets. Subjects could go ahead and respond on consistent trials, but might recheck the display on inconsistent trials, lengthening RT considerably.

There are several problems with proposing a misidentification explanation as an alternative account of the consistency effect. First, the misidentification hypothesis comes perilously close to invoking at least a borderline case of a continuous model, since it posits multiple transmissions from recognition to decision concerning a single letter. Second, it implies an exhaustive scan of the display to detect inconsistent targets, whereas other evidence suggests that visual search is self-terminating with displays of this kind (Van der Heijden, 1975; Van der Heijden \& 
Menckenberg, 1974). Third, it requires quite a few misidentifications. Assuming an extra $200 \mathrm{msec}$ is needed to recheck the display (cf. Krueger, 1978), rechecking would have to occur on $8 \%$ of all trials to account for the observed $16-\mathrm{msec}$ effect. This seems like an implausibly high percentage of recognition errors, given the good conditions of stimulus presentation.

The results of this experiment are also germaine to the issue of how recognition of one letter is influenced by the presence of a similar letter in the display. Though the stimulus materials were identical to those of Experiment 1, noise letters visually similar to the signal facilitated its recognition in Experiment 2, while they inhibited its recognition in Experiment 1 . It thus appears that the influence of a visually similar noise letter is partly determined by the potential relevance of the letter to the decision. If the noise letter appears in an irrelevant position, the act of suppressing it seems also to suppress the signal to which it is similar. If it appears in a relevant position, on the other hand, it seems to contribute positively to signal recognition. The importance of this distinction is slightly surprising with respect to previous discussions (e.g., Estes, 1974) that have emphasized structural or "hardware" interactions between letter positions.

\section{EXPERIMENT 3}

The results of the second experiment support the claim that partial activations of target letters can influence decisions under some circumstances, as predicted by continuous models. A comparison of Experiments 1 and 2 also suggests that continuous output is obtained from relevant letter positions but may not be obtained from irrelevant positions. The purpose of the next two experiments was to investigate both the generality of continuous output and the importance of relevance, using a similar task with quite different stimulus materials.

In this experiment, stimuli were large letters made out of small letters, as shown in Figure 1. Subjects were instructed to attend to letters at the local level and to ignore letters at the global level. Such stimuli have been used in many previous studies of the relationship between different levels of structure (Hoffman, 1980; Kinchla, 1974, 1977; Kinchla \& Wolfe, 1979; Martin, 1979; Miller, 1981 b; Navon, 1977; Shor, 1971). Previous work with these stimuli has demonstrated signal and response consistency effects when the signal is conveyed by the local letters and letters identical to targets are presented at the irrelevant global level (Miller, 1981b). In other words, information about the global letter is known to contribute to decisions about the local letters, even when the global letter is supposed to be ignored.

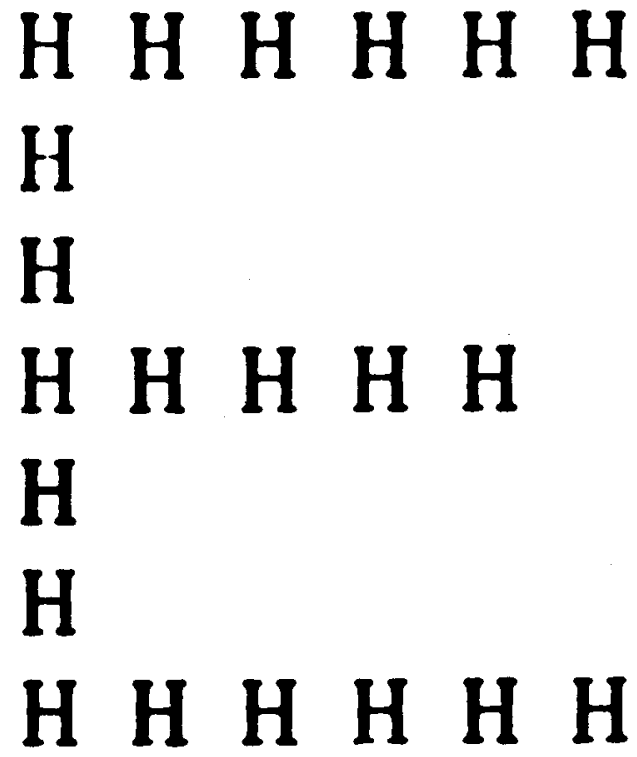

Figure 1. Sample stimulus: A large E made out of small Hs.

\section{Method}

Apparatus and Stimuli. Stimuli were presented and responses and response latencies recorded by a Terak 8510 microcomputer. Stimuli were large letter forms defined by positions of identical small letters, as shown in Figure 1. All letters were uppercase and appeared as light figures on a dark background. Each large letter was defined in terms of an array of character positions that was 11 spaces wide and 7 lines high (approximately $3.2 \times$ $3.8 \mathrm{~cm}$ ). Small letters were about $3 \mathrm{~mm}$ wide and $5 \mathrm{~mm}$ high. The stimulus displays were viewed from a distance of about $60 \mathrm{~cm}$, so the visual angles of the large and small letters were about 3 and $.3 \mathrm{deg}$, respectively. In order to equate the density of spacing for horizontal rows and vertical columns of small letters, alternate character positions were used in horizontal rows, with blanks in the unfilled positions. These stimuli were identical to those used in previously reported experiments (Miller, 1981b), and standard global precedence findings have been obtained with them (cf. Kinchla \& Wolfe, 1979). Each subject was tested alone in a small room with normal office illumination, and responses were made with the left and right index fingers on the typewriter keyboard of the Terak microcomputer.

Subjects and Procedure. Each of $\mathbf{4 8}$ right-handed undergraduates at the University of California, San Diego, participated in a single experimental session. The sessions lasted about 20-30 min, and the students partially fulfilled a requirement of an introductory psychology course through their participation.

For each subject, two target letters were assigned to each of two response keys. One target letter was selected from each of the following four visually similar letter pairs: CG, IT, UV, KR. The assignments of letters to responses and conditions were counterbalanced as completely as possible across subjects. For half of the subjects, $M$ was the neutral letter, while for the other half, it was D.

Each subject served in a single block of 282 trials. The first 30 trials were considered practice and were not recorded. Each of the four target letters was tested equally often in the remaining 252 trials. Each target letter could appear within the context of any of nine different large-letter forms: the four actual target letters, the four visually similar matches to these four target letters, or the neutral letter. Each target letter occurred equally often in each context. 
Table 3

Experiment 3: RT and PE as a Function of Noise Condition

\begin{tabular}{lll}
\hline \multicolumn{1}{c}{ Noise Condition } & RT & PE \\
\hline Neutral & 572 & 3.2 \\
ID Signal Consistent & 556 & 4.0 \\
ID Response Consistent & 567 & 3.2 \\
ID Response Inconsistent & 588 & 5.1 \\
VS Signal Consistent & 565 & 3.4 \\
VS Response Consistent & 580 & 3.2 \\
VS Response Inconsistent & 582 & 4.2 \\
\hline
\end{tabular}

Note-ID $=$ identical to target letter; $V S=$ visually similar to target letter.

\section{Results and Discussion}

For each subject, average correct RT and PE were computed as a function of the relation between the large letter and the small target. Averages of these values across subjects are shown in Table 3.

For both RT and PE, ANOVAs were computed with factors of noise condition, response hand, and subjects. No reliable effects were obtained in the analysis of PE. In the analysis of RT, the $29-\mathrm{msec}$ advantage for right-hand responses was reliable $[\mathrm{F}(1,47)=15, \mathrm{p}<.01, \mathrm{MSe}=18,640]$, as was the effect of noise condition $[F(6,282)=6.8, p<.01$, $\mathrm{MSe}=3,459$ ]. The interaction was not significant. Pairwise comparisons among the different noise conditions can be evaluated using the least significant difference of $12 \mathrm{msec}$. The finding of major interest with respect to the prediction of continuous models is that there was no difference between VS responseconsistent trials and VS response-inconsistent trials.

Finding that VS signal-consistent trials are faster than VS inconsistent trials extends previous results showing that irrelevant global letters can have an effect on responses to local letters (e.g., Navon, 1977). It is clear that letters logically irrelevant to a task can have sizable effects on performance if they are visually similar to letters that are logically relevant. However, there was no evidence of a consistency effect on VS response-consistent trials, although this effect is predicted by continuous models.

\section{EXPERIMENT 4}

Like Experiment 1, Experiment 3 seems to indicate that there is no continuous output from irrelevant stimulus channels. Again, however, it is possible that evidence for continuous output from the global letters could be obtained if the global letters were relevant to the task. This experiment used the same stimulus materials as Experiment 3, but the task was changed so that both stimulus levels were relevant.

\section{Method}

The apparatus and stimuli were the same as those used in Experiment 3. Each of 48 subjects was tested in two blocks of 160 trials, with 30 practice trials preceding the first block and 2 warm- up trials preceding the second. For each subject, two target letters were assigned to one response and two target letters were assigned to the other, as in the earlier experiments. The subjects were told that precisely one target letter would appear on each trial and that this letter could appear as either the global letter or the local letters.

The 160 experimental trials in each block were equally divided among 32 conditions logically equivalent to those in Experiment 2. There were four target letters that could appear, targets could appear at either the global or the local level, and there were four nontargets that could appear at the other level. As in Experiment 2, the nontarget was always visually similar to one of the four target letters.

\section{Results and Discussion}

The analysis paralleled that of Experiment 2, with the factor of signal level instead of signal side. For each subject and block, average correct RT and PE were computed as a function of response hand, signal level, and noise condition. Averages of these values across subjects, blocks, and response hands are shown in Table 4.

An analysis of variance of RT was carried out with factors of subjects, blocks, response hand, noise condition, and signal level. This analysis showed the reliability of the 115 -msec effect of blocks $[F(1,47)=$ $118, \mathrm{p}<.01$, $\mathrm{MSe}=31,978]$, as well as the effects of both signal level $[\mathrm{F}(1,47)=37.7, \mathrm{p}<.01, \mathrm{MSe}=$ $29,954]$ and noise condition $[F(2,94)=7.16, p<.01$, $\mathrm{MSe}=6,431]$ apparent in Table 4. The interaction displayed in the table was also reliable $[F(2,94)=$ $5.11, \mathrm{p}<.025, \mathrm{MSe}=4,820]$. The only other significant source of variation was the interaction of blocks with response hand $[\mathrm{F}(1,47)=13.6, \mathrm{p}<.01$, $\mathrm{MSe}=7,828$, arising from a bigger practice effect on the right hand than on the left. ${ }^{2}$

A planned comparison of the VS response-consistent condition with the VS response-inconsistent condition revealed that the inconsistent condition was reliably slower $(p<.05)$. This is compatible with the prediction of continuous models, and it supports the claim that stimuli may begin to influence decisions before they are fully recognized. In spite of the significant signal level $\times$ noise condition interaction, there is no basis for a claim that the two levels are asymmetric with respect to continuous output. The advantage for VS response-consistent trials over VS response-inconsistent trials was $15 \mathrm{msec}$ with targets

Table 4

Experiment 4: RT and PE as a Function of Noise Condition and Signal Level

\begin{tabular}{|c|c|c|c|c|c|c|}
\hline \multirow[b]{3}{*}{ Noise Condition } & \multicolumn{6}{|c|}{ Signal Level } \\
\hline & \multicolumn{2}{|c|}{ Global } & \multicolumn{2}{|c|}{ Local } & \multicolumn{2}{|c|}{ Average } \\
\hline & RT & PE & RT & PE & RT & $\mathrm{PE}$ \\
\hline VS Signal Consistent & 788 & 3.0 & 833 & 3.4 & 811 & 3.2 \\
\hline VS Response Consistent & 783 & 3.2 & 857 & 3.7 & 820 & 3.4 \\
\hline VS Response Inconsistent & 798 & 3.9 & 867 & 5.6 & 832 & 4.7 \\
\hline Average & 790 & 3.3 & 852 & 4.2 & & \\
\hline
\end{tabular}

Note $-V S=$ visually similar to target letter. 
at the global level and $10 \mathrm{msec}$ with targets at the local level, and these figures do not differ significantly.

The significant signal level $\times$ noise condition interaction arose because of the different pattern of results for the VS signal-consistent condition at the two levels. With a signal at the global level, a visually similar letter at the local level apparently had no effect on recognition of the global signal. However, when the signal was at the local level, recognition was greatly facilitated by a visually similar global letter. This interaction is not easily explained in terms of faster recognition of global letters than of local ones (Navon, 1977; but see Miller, 1981b). Such an explanation would also require a bigger VS responseconsistency effect with local signals than with global signals, but no such difference was found. Of course, it is possible that there really is such an effect, and that the failure to find it represents a Type II error. Alternatively, it could be that the interaction reflects an asymmetry in the combination of activation within the recognition process. For some reason, it could be easier for global letters to contribute activation to that produced by local letters than vice versa.

As in Experiment 2, finding a response-consistency effect for letters visually similar to targets confirms a prediction of continuous models. In this experiment, however, it is more plausible to argue that letters visually similar to targets may have been mistakenly recognized as targets on some proportion of the trials. Though the visual conditions of stimulus presentation were good, the perceptual task was more difficult than in Experiment 2, requiring identification of letters at two distinct levels of the visual form. Occasional misidentifications could produce very long RTs on inconsistent trials if the subject rechecked the display when two targets assigned to different responses were "recognized." According to this view, then, average RT for VS response-inconsistent trials was greater than average RT for VS responseconsistent trials because of a small percentage of especially slow responses.

In order to test the misidentification account of the VS response-consistency effect, two reanalyses of the raw RT data were performed. First, variances were computed for the five RTs in each condition within each block. If a few slow misidentification trials inflate the response-inconsistent mean, they should also inflate its variance. However, the average variance (across subjects, blocks, and letters) was slightly higher for the VS response-consistent than for the VS response-inconsistent trials. Second, the five RTs in each condition and block were rank ordered from smallest to largest. These RTs estimate the 10th, 30th, 50th, 70th, and 90th percentiles of the RT distributions from which they are drawn (cf. Ratcliff, 1979). The misidentification hypothesis predicts that the advantage for VS response-consistent trials should be greater at the higher percentiles than at the lower percentiles. When averaging the values at each per- centile across subjects, blocks, and letters, however, just the reverse was obtained. VS response-consistent trials had the biggest advantage over VS responseinconsistent trials at the 10th and 30th percentiles, and consistent trials actually took slightly longer than inconsistent trials at the 90th percentile. The effect of response inconsistency on error rates was also greater at the lower percentiles than at the higher percentiles. In the face of these analyses, the misidentification hypothesis appears unlikely.

\section{GENERAL DISCUSSION}

These studies used letters visually similar to targets in order to produce partial activation of the codes for target letters. The goal was to determine whether such partial activation would have any effect on decision processes. Decision priming by letters visually similar to targets would support continuous models, in which partial information about a stimulus is transmitted to the decision process before the stimulus is fully identified. The absence of such priming would support discrete models, in which no partial information about a letter can be sent to decision processes.

When noise letters appeared in relevant display positions, small, but statistically reliable, advantages were obtained for VS response-consistent noise letters as compared with VS response-inconsistent noise letters. This result is easily explained by models allowing continuous transmission of information from the recognition process to the decision process. The noise letters are assumed to produce partial activations of the target letters to which they are similar, and these partial activations are transmitted to the decision process during which they contribute activation either to the same response as the signal letter (VS response consistent) or to the opposite response (VS response inconsistent).

Interestingly, the response-consistency effect for visually similar letters was not obtained with noise letters in irrelevant positions. To the extent that this particular consistency effect is regarded as an important test of continuous output, the null finding suggests that information about irrelevant positions is not transmitted continuously. It is quite possible, of course, that effects of continuous output from these sources were simply too small to be detected. Information from irrelevant sources seems to be attenuated somewhat (e.g., Treisman, 1964), and attenuation might simply make it very difficult to detect effects that are rather small anyway. This is certainly the most parsimonious conclusion that can be drawn from the results, in view of the evidence for continuous output from relevant information sources. Another possibility, though, is that discrete thresholds can be set up to help suppress information from irrelevant sources. One could argue that attenuation should not have been severe enough to elimi- 
nate the effect entirely, especially since response consistency effects were always obtained when letters identical to targets appeared in the irrelevant positions. The present results are not sufficient to discriminate between these two positions.

Though this article has emphasized the test of a particular consistency effect predicted by continuous models, it is important to consider the extent to which the various kinds of consistency effects are incompatible with discrete models. For example, previous findings of consistency effects for noise letters identical to target letters can easily be explained by discrete models in terms of decision-level competition between fully recognized signal and noise letters. The decision process is often unable to ignore irrelevant information (e.g., C. W. Eriksen \& Hoffman, 1972, 1973; Logan, 1980; Stroop, 1935), and noise letters might contribute some activation to the decision because of imperfect selection. This activation could speed decisions on consistent trials while slowing them on inconsistent trials.

C. W. Eriksen and Schultz (1979) also reported that consistency effects were sensitive to a variety of variables controlling the relative speed of recognizing signal and noise letters, such as relative size and contrast of signal and noise letters. Consistency effects were large to the extent that noise letters were bigger or higher in contrast than signal letters. Furthermore, variations in target-noise SOA influenced consistency effects, with effects tending to be larger when the noise letters appeared slightly ahead of the signal. In order to reconcile discrete models with these results, it need only be assumed that these variables influence the distributions of random relative completion times for recognizing signal and noise letters. A noise letter would produce a consistency effect only on trials in which it was recognized within a certain period immediately preceding the recognition of the signal. Then variables that control the relative completion times of signal and noise letters would modulate the size of consistency effects. If the distributions of completion times were fairly wide and overlapping, this modulation could appear to be quite gradual, as if it resulted from an effect on a continuous variable, such as strength of activation.

It is more difficult for discrete models to account for B. A. Eriksen and C. W. Eriksen's (1974) finding that VS signal-consistent noise letters facilitate responses, while VS response-inconsistent noise letters inhibit responses. Discrete models cannot easily explain this effect in the decision process, since the noise letters were not identical to, but merely visually similar to, target letters. These noise letters should have had no influence on the decision process after they were completely recognized, since the noise letters did not themselves belong to either target set. If no information about a noise letters is transmitted to the decision process until the letter has been fully identified, as claimed by discrete models, then noise letters belonging to neither target set should have no effect on the decision. ${ }^{3}$ However, it is possible to explain this result with a discrete model in which the effect occurs during recognition rather than during decision. Noise letters similar to the signal could contribute activation in support of the signal letter, which would speed recognition of that signal letter (cf. Logan, 1980). Noise letters similar to targets other than the signal could draw attention away from the signal letter, thus slowing its recognition. (cf. Duncan, 1980).

Of the various consistency effects, it seems that the one most difficult to explain with a discrete model is the VS response-consistency effect obtained in Experiments 2 and 4 . In the present VS response-consistent and response-inconsistent conditions, noise letters were not actually target letters. Therefore, it is difficult to argue that they would have any biasing effect on decisions once they were fully recognized. This implies that their effect arises before they are fully recognized. Furthermore, in both the VS responseconsistent condition and the VS response-inconsistent condition, the noise letters were similar to a target letter other than the signal. This makes it difficult to explain the effect within recognition, because the two different classes of noise letters seem to be equated with respect to their effects on that process. According to the discrete explanation of the VS signal-consistent advantage and the VS responseinconsistent disadvantage outlined above, both response-consistent and response-inconsistent noise letters should draw attention away from the signal letter and thus inhibit its recognition. Thus, it is quite difficult for discrete models to predict a difference between trials with VS response-consistent noise letters and trials with VS response-inconsistent noise letters.

The question of whether transmission of information from the recognition to the decision process is continuous or discrete is bound to be a very complicated one. It seems likely that the answer to this question will depend on many factors, including the nature of the task and the nature of the stimulus set. Given that some evidence of continuous output has been found, however, it is important not to overgeneralize the conclusions drawn from these effects. Certainly this is clear from the suggestion of a difference between processing of relevant and irrelevant information sources. More importantly, however, the present results must be regarded as evidence only of continuous output from the recognition process to the decision process. Other experiments using similar criteria (Miller, 1981a) have explicitly sought and failed to find comparable evidence of response activation based on continuous output from the decision process. Again, this failure may reflect lack of power in the experimental design. Alternatively, however, it is logically possible that transmission of information from recognition to decision processes could be con- 
tinuous while transmission from decision to response activation could be discrete. The conclusions of the present work, therefore, must at present be limited to the transmission of information from recognition to decision processes.

\section{REFERENCES}

Bjork, E., \& Murray, J. On the nature of input channels in visual processing. Psychological Review, 1977, 84, 472-484.

Brondbent, D. Perception and communication. New York: Pergamon Press, 1958.

Donders, F. Die Schnelligkeit psychischer Processe. Archiv Anatomie und Physiologie, 1868, 657-681.

Duncan, J. The locus of interference in the perception of simultaneous stimuli. Psychological Review, 1980, 87, 272-300.

Eriksen, B. A., \& Eniksen, C. W. Effects of noise letters upon the identification of a target letter in a nonsearch task. Perception \& Psychophysics, 1974, 16, 143-149.

ERIKSEN, C. W. Independence of successive inputs and uncorrelated error in visual form perception. Journal of Experimental Psychology, 1966, 72, 26-35.

Eriksen, C. W., \& Eriksen, B. A. Target redundancy in visual search: Do repetitions of the target within the display impair processing? Perception \& Psychophysics, 1979, 26, 195-205.

Eriksen, C. W., \& Hoffman, J. E. Temporal and spatial characteristics of selective encoding from visual displays. Perception \& Psychophysics, 1972, 12, 201-204.

Eriksen, C. W., \& Horrman, J. E. The extent of processing of noise elements during selective encoding from visual displays. Perception \& Psychophysics, 1973, 14, 155-160.

Eriksen, C. W., \& LApPin, J. S. Internal perceptual system noise and redundancy in simultaneous inputs in form identification. Psychonomic Science, 1965, 2, 351-352.

ERIKsen, C. W., \& Schultz, D. W. Information processing in visual search: A continuous flow conception and experimental results. Perception \& Psychophysics, 1979, 25, 249-263.

EstEs, W. K. Redundancy of noise elements and signals in visual detection of letters. Perception \& Psychophysics, 1974, 16, 53-60.

HofFMAN, J. Interaction between global and local levels of a form. Journal of Experimental Psychology: Human Perception and Performance, 1980, 6, 222-234.

KINCHLA, R. A. Detecting target elements in multielement arrays: A confusability model. Perception \& Psychophysics, 1974, 15, 149-158.

Kinchla, R. A. The role of structural redundancy in the perception of visual targets. Perception \& Psychophysics, 1977, 22, 19-30.

Kinchla, R. A., \& Wolfe, J. M. The order of visual processing: "Top-down," "bottom-up," or "middle-out." Perception \& Psychophysics, 1979, 25, 225-231.

KRUeger, L. E. A theory of perceptual matching. Psychological Review, 1978, 85, 278-304.

Krueger, L. E., \& Shapiro, R. G. Repeating the target neither speeds nor slows its detection: Evidence for independent channels in letter processing. Perception \& Psychophysics, 1980, 28, 68-76.

LogAN, G. Attention and automaticity in Stroop and priming tasks: Theory and data. Cognitive Psychology, 1980, 12, 523-553.

MArtin, M. Local and global processing: The role of sparsity. Memory \& Cognition, 1979, 7, 476-484.

MCClelland, J., \& Rumelhart, D. An interactive activation model of context effects in letter perception. Part I: An account of basic findings. Psychological Review, 1981, 88, 375-407.

Mewhort, D. J. K., Campbele, A. J., Marchetti, F. M., \& CAMPBELL, J. I. D. Identification, localization, and "iconic memory": An evaluation of the bar-probe task. Memory \& Cognition, 1981, 9, 50-67.

Millen, J. Discrete vs. continuous stage models of human information processing: In search of partial output. Journal of
Experimental Psychology: Human Perception and Performance, 1981 , in press. (a)

Miller, J. Global precedence in attention and decision. Journal of Experimental Psychology: Human Perception and Performance, 1981, 7, 1161-1174. (b)

Millen, J., \& BAUER, D. Visual similarity and discrimination demands. Journal of Experimental Psychology: General, 1981, 110, 39-55.

Miller, J., \& Hardzinski, M. Case specificity of the stimulus probability effect. Memory \& Cognition, 1981, 9, 205-216.

Navon, D. Forest before trees: The precedence of global features in visual perception. Cognitive Psychology, 1977, 9, 353-383.

Norman, D., \& Boвrow, D. On data-limited and resourcelimited processes. Cognitive Psychology, 1975, 7, 44-64.

RATCLIFF, R. Group reaction time distributions and an analysis of distribution statistics. Psychological Bulletin, 1979, 86, 446-461.

Santee, J., \& Egeth, H. Interference in letter identification: A test of feature-specific inhibition. Perception \& Psychophysics, $1980,27,321-330$.

Sнor, R. Symbol processing speed differences and symbol interference effects in a variety of concept domains. Journal of General Psychology, 1971, 85, 187-205.

Simon, J. Reactions toward the source of stimulation. Journal of Experimental Psychology, 1969, 81, 174-176.

Smith, E. Choice reaction time: An analysis of the major theoretical positions. Psychological Bulletin, 1968, 69, 77-110.

SPE RLING, G. Successive approximations to a model for shortterm memory. Acta Psychologica, 1967, 27, 285-292.

SternBerg, S. Memory scanning: Mental processes revealed by reaction-time experiments. American Scientist, 1969, 57, 421457. (a)

Sternaerg, S. The discovery of processing stages: Extensions of Donders' method. Acta Psychologica, 1969, 30, 276-315. (b)

STroop, J. Studies of interference in serial verbal reactions. Journal of Experimental Psychology, 1935, 18, 643-662.

Treisman, A. Verbal cues, language and meaning in selective attention. American Journal of Psychology, 1964, 77, 206-219.

VAN DER HeiJden, A. Some evidence for a limited capacity parallel self-terminating process in simple visual search tasks. Acta Psychologica, 1975, 39, 21-41.

Van der Heijden, A., \& Menckenberg, H. Some evidence for a self-terminating process in simple visual search tasks. Acta Psychologica, 1974, 38, 169-181.

\section{NOTES}

1. Consideration of how discrete models might also account for these results will be deferred to the discussion section.

2. Subjects were initially told the target letters for the left hand, followed by the target letters for the right hand. This probably led to faster learning of the left target set, so that practice had less effect on that set.

3. As an anonymous reviewer pointed out, this argument assumes that the final output of the identification process activates only the presented letter. If the result of identification were to activate all letter codes in proportion to their visual similarity to the presented letter, then discrete models would also predict consistency effects for letters similar to targets. Such a model is implausible on theoretical grounds, however. Even in continuous models (e.g., McClelland \& Rumelhart, 1981), identification processes are generally assumed to result primarily in activation of one dominant code, with activation of codes for similar items returning to comparatively low levels as identification reaches asymptote. Furthermore, there is a variety of evidence that the output of the letter-identification process is an abstract code not representing the visual features of the input letter (see Miller \& Hardzinski, 1981, for a review).

(Manuscript received June 18, 1981; revision accepted for publication November 2,1981 .) 